\title{
Carcinoma Microinvasor no Cone Pós Biópsia Dirigida Compativel com NIC 3
}

\author{
Microinvasive Carcinoma in the Cone Specimen in Women with Colposcopically \\ Directed Biopsy Suggesting CIN 3
}

Priscila Garcia Figueiredo, Renata Clementino Gontijo, Sophie Françoise Mauricette Derchain Fabiana Yumi Nakano, Julio César Teixeira, Edson Zangiacomi Martinez

\section{RESUMO}

Objetivo: determinar os fatores associados ao achado de carcinoma microinvasor no cone de mulheres com biópsia colpodirigida prévia compativel com neoplasia intra-epitelial cervical (NIC) 3 e avaliar a proporção de margens comprometidas.

Pacientes e métodos: foram revisados os prontuários de 385 mulheres (média de idade: 39 anos) submetidas à conização a frio ou por cirurgia de alta freqüência (CAF) com alça no periodo de janeiro de 1993 a julho de 2000. Estes procedimentos foram indicados por biópsia compativel com NIC 3.

Resultados: o diagnóstico do cone foi compativel com NIC 3 em 243 mulheres (63\%) e com NIC 2 em 13 (3\%). Apenas 10 apresentaram HPV/NIC 1 (3\%) e oito não tinham doença residual no cone. Entretanto, 101 mulheres apresentaram carcinoma microinvasor no cone (26\%) $e$ $10(3 \%)$ carcinoma invasor franco. A idade, o estado menstrual e o número de partos não estiveram relacionados com a gravidade da lesão no cone. Mulheres com alterações da colpocitologia oncológica sugestivas de invasão apresentaram um risco significativamente maior de apresentar carcinoma microinvasor ou invasor no histológico final ( $p<0,01)$, embora 52 das 243 mulheres com NIC 2 ou NIC 3 no cone também tivessem sugestão de invasão na colpocitologia. Entre as mulheres com NIC 2 ou 3, 44\% apresentaram epitélio branco, 21\% pontilhado e $17 \%$ mosaico. Esta proporção foi semelhante nas mulheres com carcinoma microinvasor ou invasor, sendo estas imagens encontradas, respectivamente, em 37\%, 23\% e 21\%. O comprometimento das margens do cone foi significativamente maior nas mulheres submetidas a CAF (49\%) do que naquelas submetidas à conização a frio (29\%).

Conclusão: a ausência de fatores independentes clínicos e colposcópicos que se associam com o achado de carcinomas microinvasivos em mulheres submetidas à conização por biópsia compativel com NIC 3 justifica a excisão cônica da junção escamo-colunar nas lesões cervicais de alto grau.

PALAVRAS-CHAVE: Conização. Colposcopia. Neoplasia intra-epitelial. Carcinoma microinvasor.

Departamento de Tocoginecologia da Faculdade de Ciências Médicas e Centro de Atenção Integral à Saúde da Mulher (CAISM) da Universidade Estadual de Campinas (UNICAMP) Correspondência:

Sophie F. M. Derchain

Rua Antonio Hossri, 629 - Cidade Universitária

13083-370 - Campinas - SP

Fone: (19) 3788-9305/ Fax: (19) 32895935

e-mail: derchain@supernet.com.br

Orgãos financiadores: FAPESP (00/05644-3) e CNPq (300354/01)

\section{Introdução}

Nos últimos trinta anos, diante de um resultado alterado da colpocitologia oncológica ( $\mathrm{CO})$, a avaliação colposcópica com biópsia dirigida das áreas anormais vem sendo usada como padrão diagnóstico para determinar a gravidade da lesão cervical, antes de se iniciar o tratamento ${ }^{1,2}$. O desempenho da colposcopia em identificar áreas com lesões mais graves tem sido questionado, e 
pode existir proporção significativa de mulheres com neoplasia intra-epitelial cervical (NIC) 3 na ectocérvice e lesão endocervical mais grave não evidenciada na colposcopia. Conseqüentemente, o resultado de biópsia orientada por colposcopia pode subestimar a gravidade da lesão, levando a tratamento inadequado e com risco de persistência da doença ou desenvolvimento de câncer. $\mathrm{Na}$ avaliação histopatológica do cone pode ser encontrada doença invasora ou microinvasora não diagnosticada na biópsia em mais de $10 \%$ dos $\operatorname{casos}^{3,4}$.

Assim, uma vez detectada uma NIC 3 pela biópsia, a conização é o tratamento preferencial, pois permite avaliação histológica completa de toda a zona de transformação. Até há poucos anos a conização era realizada com bisturi a frio em centro cirúrgico. Atualmente, a cirurgia de alta freqüência com alça (CAF), método introduzido por Prendiville et al. ${ }^{3}$, vem sendo cada vez mais utilizada para a excisão de toda a zona de transformação ${ }^{1,2,4,5}$. Inicialmente a conização com CAF era reservada para os casos em que a zona de transformação era totalmente visivel e confinada ao colo e quando não havia suspeita de doença microinvasora ou invasora na citologia e na colposcopia ${ }^{3}$. Porém, tem sido demonstrado que a conização por CAF apresenta resultados terapêuticos similares à conização a frio e é um método mais rápido, mais econômico e com menos complicações ${ }^{6}$.

A freqüência de comprometimento das margens do cone varia de 7,2 a $43,5 \%$ e depende de vários fatores, tais como grau de gravidade da NIC, envolvimento da endocérvice, dimensões do cone e técnica de conização. Sabe-se hoje que uma conização ampla e com adequada avaliação das margens cirúrgicas é suficiente para o tratamento da NIC 3 e até do carcinoma microinvasor Ia $1^{7}$. A presença de recidiva ou persistência da doença após conização está significativamente relacionada ao comprometimento das margens, podendo ser observada em 30\% das mulheres com margens comprometidas. Entretanto, 28\% das mulheres com margens próximas ou com artefatos térmicos e $8 \%$ daquelas com margens livres também apresentam recidiva ${ }^{8,9}$.

Assim, o objetivo deste estudo foi determinar os fatores associados ao achado de carcinoma microinvasor em mulheres submetidas à conização por biópsia colpodirigida prévia compatível com NIC 3, e avaliar a proporção de margens comprometidas em relação ao tipo de conização realizada.

\section{Pacientes e Métodos}

Foram avaliados os prontuários de $625 \mathrm{mu}-$ lheres submetidas à conização no Centro de Atenção Integral à Saúde da Mulher (CAISM) da Universidade Estadual de Campinas (UNICAMP), de janeiro de 1993 a julho de 2000, após aprovação do projeto pelo Comitê de Ética em Pesquisa da Faculdade de Ciências Médicas da UNICAMP. Foram selecionadas para este estudo 385 mulheres com biópsia dirigida por colposcopia compativel com NIC 3 realizada previamente à conização. A média de idade foi de 39 anos (mínima de 17 e máxima de 80 anos). Todas as mulheres incluídas no estudo tinham sido avaliadas no Ambulatório de Patologia Cervical após serem encaminhadas por alterações morfológicas na $\mathrm{CO}$, colposcopia atipica ou biópsia sugestiva de NIC 3. Destas, 351 foram submetidas à coleta de nova $\mathrm{CO}$ e todas as lâminas citológicas foram avaliadas pelo Laboratório de Citopatologia do CAISM e interpretadas como: esfregaço dentro do limite da normalidade, sugestivo de ASCUS, HPV, NIC 1, NIC 2, NIC 3, carcinoma escamoso microinvasor ou invasor e lesões glandulares. Todas as mulheres realizaram colposcopia com aplicação de ácido acético a 2 ou $3 \%$. A colposcopia foi classificada em satisfatória ou insatisfatória, em relação à visualização da junção escamo-colunar. Nos casos satisfatórios a imagem colposcópica foi classificada em normal e anormal, com presença ou ausência de epitélio branco, mosaico, vasos atípicos, pontilhado e leucoplasia. Em relação à técnica, 303 mulheres foram submetidas à conização a frio (79\%) e 82 foram submetidas à conização por CAF (21\%). A conização a frio foi realizada no Centro Cirúrgico do CAISM-UNICAMP sob anestesia geral, raquidiana ou peridural, conforme a técnica clássica, e sutura com pontos de Sturmdorf modificados. A conização por CAF foi realizada no Ambulatório de Patologia Cervical do CAISM, por meio da utilização de um gerador para cirurgia de alta freqüência (modelo SS-200 da WEM) acoplado a um aspirador de vapores com filtro biológico (Wavevac, da Loktal), sob bloqueio anestésico com infiltração de $0,4 \mathrm{ml}$ de cloridrato de lidocaína a $2 \%$, com norepinefrina 1:50.000 nos quatro pontos cardeais do colo uterino. Após a conização era realizada a hemostasia das bordas por cauterização com um eletrodo esférico de $5 \mathrm{~mm}$ e aplicação da solução de Monsel.

Os espécimes da biópsia dirigida e da conização a foram incluídos em parafina e as lâminas foram coradas pelo método de hematoxilinaeosina. Foram realizadas de 15 a 20 lâminas por cone, três a cinco por quadrante, e avaliados os 
graus da lesão e comprometimento das margens. Adotou-se a classificação da Organização Mundial da Saúde ${ }^{10}$ para catalogar os resultados em: cervicite, condiloma e NIC 1, agrupados em HPV/ NIC 1, NIC 2 e NIC 3, carcinoma microinvasor e carcinoma invasor. A análise dos dados foi realizada pelo teste exato de Fisher e utilizou-se o programa de computador versão SAS 8.0.

\section{Resultados}

O diagnóstico do cone foi compativel com NIC 3 em 243 mulheres (63\%) e com NIC 2 em 13 (3\%). Apenas 10 apresentaram HPV/NIC 1 (3\%) e oito não tinham doença residual no cone. Entretanto, 101 mulheres apresentaram carcinoma microinvasor $(26 \%)$ e $10(3 \%)$, carcinoma invasor franco (Tabela 1).
Tabela 1 - Distribuição das mulheres com biópsia dirigida pela colposcopia compativel com NIC 3, segundo o diagnóstico histológico do cone.

\begin{tabular}{lrr}
\hline Diagnóstico histológico do cone & $\mathbf{n}$ & \% \\
\hline Cervicite & 8 & 2 \\
HPV/NIC 1 & 10 & 3 \\
NIC 2 & 13 & 3 \\
NIC 3 & 243 & 63 \\
Microinvasor & 101 & 26 \\
Invasor & 10 & 3 \\
Total & 385 & 100 \\
\hline
\end{tabular}

Na Tabela 2 observamos que das 111 mulheres com carcinoma microinvasor ou invasor no cone, $71(64 \%)$ tinham idade inferior a 45 anos, e entre as 243 mulheres que apresentaram NIC 2 ou 3, 183 $(71 \%)$ tinham menos de 45 anos $(\mathrm{p}=0,17)$. O estado menstrual e o número de partos também não estiveram relacionados com a gravidade da lesão.

Tabela 2 - Distribuição das mulheres segundo a idade, estado menstrual e paridade e o diagnóstico histológico do cone.

\begin{tabular}{|c|c|c|c|c|c|c|c|c|}
\hline \multirow[t]{2}{*}{$\begin{array}{l}\text { Fatores } \\
\text { associados }\end{array}$} & \multicolumn{2}{|c|}{$\begin{array}{l}\text { Cervicite, } \\
\text { HPV, NIC } 1\end{array}$} & \multicolumn{2}{|c|}{ NIC 2 ou 3} & \multicolumn{2}{|c|}{$\begin{array}{l}\text { Microinvasor ou } \\
\text { invasor }\end{array}$} & \multirow[b]{2}{*}{ Total } & \multirow[b]{2}{*}{$\mathrm{p}^{*}$} \\
\hline & $n$ & $(\%)$ & $\mathrm{n}$ & $(\%)$ & $\mathrm{n}$ & $(\%)$ & & \\
\hline \multicolumn{9}{|l|}{ Idade } \\
\hline$<45$ anos & 15 & (83) & 183 & (71) & 71 & (64) & 269 & \\
\hline$>45$ anos & 3 & (17) & 73 & (29) & 40 & (36) & 116 & 0,17 \\
\hline \multicolumn{9}{|l|}{ Menopausa } \\
\hline Sim & 4 & (22) & 52 & (20) & 29 & (26) & 85 & \\
\hline Não & 14 & (78) & 204 & (80) & 82 & (74) & 300 & 0,45 \\
\hline \multicolumn{9}{|l|}{ Parto } \\
\hline Até 2 & 6 & (33) & 108 & (42) & 42 & (38) & 156 & \\
\hline 3 ou mais & 12 & (66) & 148 & (58) & 69 & (62) & 229 & 0,62 \\
\hline Total & 18 & & 243 & & 111 & & 385 & \\
\hline
\end{tabular}

* teste exato de Fisher

Na Tabela 3, avaliamos a correlação entre o resultado da CO e do cone. Das 256 mulheres NIC 2 ou NIC 3 no espécime cirúrgico, 185 (72\%) apresentavam alterações morfológicas de alto grau (sugestivas de NIC 2 ou NIC 3) na CO, enquanto que
$52(20 \%)$ apresentavam alterações sugestivas de invasão. Por outro lado, entre as 109 mulheres com microinvasão ou invasão no cone, 45 (41\%) tinham alterações sugestivas de microinvasão ou invasão na $\mathrm{CO}(\mathrm{p}<0,01)$.

Tabela 3 - Distribuição das mulheres segundo o resultado da citologia oncológica e o diagnóstico histológico do cone.

\begin{tabular}{|c|c|c|c|c|c|c|c|}
\hline \multirow{3}{*}{ Citologia } & \multicolumn{6}{|c|}{ Resultado histológico do cone } & \multirow{3}{*}{ Total } \\
\hline & \multicolumn{2}{|c|}{ Cerv/HPV/NIC 1} & \multicolumn{2}{|c|}{ NIC 2 ou 3} & \multicolumn{2}{|c|}{ Microinvasor ou Invasor } & \\
\hline & $\mathrm{n}$ & $(\%)$ & $\mathrm{n}$ & $(\%)$ & $\mathrm{n}$ & $(\%)$ & \\
\hline Infecção HPV, NIC 1 & 2 & (11) & 19 & (8) & 8 & (7) & 29 \\
\hline NIC 2 ou 3 & 14 & $(78)$ & 185 & $(72)$ & 56 & $(52)$ & 255 \\
\hline Microinvasor ou invasor & 2 & $(11)$ & 52 & $(20)$ & 45 & (41) & 99 \\
\hline Total & 18 & & 256 & & $109^{*}$ & & 383 \\
\hline
\end{tabular}

$p<0,001$ (teste exato de Fisher)

${ }^{*} 2$ casos sem citologia 
Na Tabela 4, comparamos o resultado da colposcopia com o resultado histológico do cone. Apenas 15 mulheres apresentaram colposcopia normal. Entre as 256 mulheres com NIC 2 ou 3, $44 \%$ apresentaram epitélio branco, 21\% apresentaram pontilhado e $17 \%$ mostraram mosaico. Esta proporção foi semelhante nas 111 mulheres com carcinoma microinvasor ou invasor, nas quais o epitélio branco foi encontrado em $37 \%$, o ponti- lhado em $23 \%$ e o mosaico em $21 \%$. A leucoplasia e vasos atípicos foram encontrados em apenas 19 e 70 casos, respectivamente, e sua distribuição foi semelhante entre mulheres com NIC 2 ou 3 ou carcinoma microinvasor e invasor. Portanto, nesta casuística, não se observou nenhuma imagem colposcópica específica para diferenciar carcinoma microinvasor ou invasor.

Tabela 4 - Distribuição das mulheres segundo o resultado da colposcopia e o diagnóstico histológico do cone.

\begin{tabular}{|c|c|c|c|c|c|c|c|}
\hline \multirow{3}{*}{ Colposcopia } & \multicolumn{6}{|c|}{ Resultado histológico do cone } & \multirow{3}{*}{ Total $^{*}$} \\
\hline & \multicolumn{2}{|c|}{ Cerv/HPV/NIC 1} & \multicolumn{2}{|c|}{ NIC 2 ou 3} & \multicolumn{2}{|c|}{ Micoinvasor ou invasor } & \\
\hline & $\mathrm{n}$ & $(\%)$ & $\mathrm{n}$ & $(\%)$ & $\mathrm{n}$ & $(\%)$ & \\
\hline Normal & 1 & (4) & 11 & (3) & 3 & (1) & 15 \\
\hline Epitélio branco & 9 & (38) & 160 & (44) & 63 & (37) & 232 \\
\hline Pontilhado & 6 & (25) & 77 & (21) & 39 & (23) & 122 \\
\hline Mosaico & 5 & (21) & 64 & (17) & 36 & (21) & 105 \\
\hline Leucoplasia & 0 & $(0)$ & 16 & (4) & 3 & (2) & 19 \\
\hline Vasos atípicos & 3 & (12) & 39 & (11) & 28 & (16) & 70 \\
\hline
\end{tabular}

* Há mulheres com mais de um achado colposcópico

Na Tabela 5 avaliamos a presença de doença nas margens dos cones, segundo a técnica utilizada: conização a frio e por CAF. Observamos que a percentagem de margens comprometidas nas mulheres submetidas à conização por CAF foi de $49 \%$, sendo este comprometimento significativamente menor $(\mathrm{p}<0,01)$ nas mulheres submetidas à conização a frio $(29 \%)$.

Tabela 5 - Distribuição das mulheres segundo a técnica da conização, o diagnóstico histológico e o comprometimento das margens.

\begin{tabular}{|c|c|c|c|c|}
\hline \multirow[b]{2}{*}{ Técnica do cone } & \multicolumn{3}{|c|}{ Diagnóstico histológico do cone } & \multirow[b]{2}{*}{$\begin{array}{c}\text { Total } \\
\text { MC/ML (\%) }\end{array}$} \\
\hline & $\begin{array}{c}\text { Cerv/HPV/NIC } 1 \\
\text { MC/ML (\%) }\end{array}$ & $\begin{array}{l}\text { NIC } 2 \text { ou } 3 \\
\text { MC/ML }(\%)\end{array}$ & $\begin{array}{l}\text { Microinvasor ou invasor } \\
\text { MC/ML (\%) }\end{array}$ & \\
\hline Conização a frio $(n=303)$ & $1 / 14$ & $45 / 151(23)$ & $41 / 52$ & $86 / 217(29)$ \\
\hline $\operatorname{CAF}(n=82)$ & $(25)$ & $29 / 31 \quad(49)$ & $10 / 8 \quad(55)$ & $40 / 42 \quad(49)$ \\
\hline
\end{tabular}

\section{Discussão}

O resultado do cone foi concordante com o diagnóstico da biópsia de NIC 3 em 243 mulheres (63\%). A taxa de concordância entre a biópsia colposcopicamente dirigida e a conização descritas na literatura varia de 50 a 93\%, sendo esta taxa maior nas lesões intra-epiteliais de alto grau e naquelas com suspeita de invasão franca ${ }^{11}$. A literatura tem mostrado que a biópsia não é padrão diagnóstico adequado para selecionar o tratamento. Ehsani et al. ${ }^{12}$, após avaliarem mulheres submetidas à CAF por citologia compatível com NIC 2 e 3 e resultado de biópsia sugestivo de NIC 1, encontraram lesão de alto grau em $41 \%$ dos cones, indicando o resultado falso-negativo da biópsia. Buxton et al. ${ }^{13}$, avaliando 243 mulheres submetidas à conização e com citologias com alterações celulares graves, encontraram discordância em $54 \%$, e com lesão mais grave no cone, em $47 \%$ dos casos, incluindo três adenocarcinomas in situ e um caso de câncer invasor.

Observamos em nosso estudo que $28,8 \%$ das mulheres com achado de NIC 3 na biópsia apresentaram carcinoma microinvasor ou invasor no cone. Isto ilustra a limitação da biópsia dirigida por colposcopia na detecção do carcinoma micro- 
invasor ou com invasão mínima. A taxa de microinvasão descrita na literatura em mulheres submetidas à conização por biópsia compatível com NIC 3 varia de 0,8 a 3\% $\%^{1,2,14}$. Entretanto, segundo Baldauf et al. ${ }^{14}$, quando se detecta um carcinoma microinvasor no cone, a sensibilidade da biópsia é de 31\%. Estes autores relatam que, dos 16 carcinomas microinvasores detectados pelo cone entre seus casos, 11 apresentavam biópsia compatível com NIC 3. Orlandi et al. ${ }^{15}$ observaram que, de 70 mulheres com carcinoma microinvasor diagnosticado nas peças dos cones ou histerectomias (todas com biópsia prévia), somente em 40\% havia sido evidenciada microinvasão na biópsia, enquanto que os $60 \%$ restantes foram subestimados. Sugerem que a biópsia não é ideal para o diagnóstico de carcinoma microinvasor inicial, pois a pequena amostra de tecido não pode evidenciar a presença de invasão estromal nem sua profundidade. A taxa de resultados falso-negativos da biópsia foi de 25\% e de carcinoma microinvasor foi de $1 \%$. Em recente estudo nacional, Reis et al. ${ }^{16}$ demonstraram a dificuldade em se predizer pela biópsia a invasão subclínica em mulheres com câncer inicial: observaram que em 113 casos de câncer invasor a sensibilidade da biópsia dirigida pela colposcopia foi de $59 \%$.

Em nosso estudo, embora a CO tenha sido um fator significativamente associado à gravidade da lesão no cone, apenas $41 \%$ das mulheres com microinvasão ou invasão apresentaram alterações citológicas compatíveis com estes diagnósticos. Por outro lado, cerca de $20 \%$ das mulheres cujo diagnóstico final foi compativel com NIC 2 ou NIC 3 também apresentaram suspeita de invasão na citologia. A CO é o método mais difundido mundialmente para rastreamento das lesões precursoras do câncer cervical, porém não pode ser considerada preditiva da gravidade exata da lesão ${ }^{17}$.

A colposcopia não foi um bom fator preditivo da gravidade da lesão cervical e tende a subestimar o carcinoma microinvasor, pois as imagens, além de não serem características, são dificeis de interpretar e as lesões microinvasoras freqüentemente são endocervicais. Os sinais colposcópicos são ainda mais inconsistentes quando o foco de invasão é pequeno e superficial. Choo et al. ${ }^{18}$ observam que não existem achados colposcópicos específicos para o carcinoma microinvasor, o que também evidenciamos em nossa casuística, na qual os achados colposcópicos foram semelhantes nos casos com NIC 3 e carcinoma microinvasor ou invasor. Hopman et al. ${ }^{11}$ concluem que a colposcopia mostra melhor desempenho em identificar doença pré-maligna ou francamente invasora do que lesões microinvasoras.

Foi muito discutida na década de 80 a reali- zação de biópsias múltiplas para o diagnóstico préoperatório de invasão focal ${ }^{19}$. Entretanto, esta conduta tem sido questionada, pois a excisão de toda a zona de transformação, atualmente realizada com custo baixo e pequeno desconforto pela cirurgia de alta freqüência, permite uma melhor acurácia diagnóstica e provou ser tratamento efetivo para as NIC, com baixa morbidade e com minimos efeitos a longo prazo sobre a menstruação e a fertilidade ${ }^{3,4,11}$.

Em nosso estudo observamos que as margens estavam comprometidas em $28 \%$ dos cones obtidos a frio e $49 \%$ dos cones obtidos por CAF. Em outra publicação brasileira, Carvalho ${ }^{20}$ estudou 154 mulheres com biópsias compativeis com NIC 3 e NIC 3 com suspeita de invasão, diagnosticadas por amputação do colo (41), conização a frio (60) e conização por CAF (53). As taxas de comprometimento das margens foram de 19,5\%, 46,7\% e $61,5 \%$, respectivamente. Este resultados foram concordantes com nosso estudo, em que encontramos o dobro da freqüência de margens comprometidas nas conizações por CAF em comparação a conização a frio. Existem controvérsias em relação ao desempenho da conização CAF na determinação das margens, devido à maior freqüência de artefatos térmicos da técnica. A alça leva à coagulação do tecido, o que dificulta a interpretação histológica, porém o dano térmico depende muito da prática e experiência do cirurgião. Taha et al. ${ }^{21}$ verificaram que, apesar de o dano térmico ter ocorrido em todos os casos, em 91\% das vezes ele foi insignificante e não prejudicou a avaliação histopatológica correta.

Existe hoje uma controvérsia em relação à conduta a ser tomada frente a uma mulher submetida à excisão da NIC e com margens comprometidas $^{8,22}$. Classicamente, mulheres cujo resultado do espécime cirúrgico apresenta neoplasia intra-epitelial são consideradas tratadas quando as margens estão livres, embora cerca de $8 \%$ apresentem doença residual quando submetidas a novo procedimento e cerca de 4 a $17 \%$ recidivem quando submetidas a seguimento ${ }^{14}$. Quando as margens do espécime estão comprometidas, a conduta posterior é controversa, visto que $70 \%$ das mulheres não têm doença residual na peça cirúrgica do segundo procedimento. Atualmente, a maioria dos autores concorda que a excisão incompleta da NIC após uma excisão com alça larga da zona de transformação merece um seguimento cuidadoso, mas não um retratamento.

Em relação ao carcinoma microinvasor Ia1, o tratamento conservador tem sido cada vez mais aceito. Entretanto, a conização somente é considerada curativa se a área inteira de lesão for removida e se as margens forem corretamente ava- 
liadas ${ }^{23,24}$. Quando as margens estão comprometidas, é obrigatório um segundo procedimento diagnóstico: nova conização ou histerectomia. Em relação à técnica de conização, Tseng et al. ${ }^{23}$ avaliaram mulheres com carcinoma microinvasor submetidas à conização por CAF e concluíram que o tratamento é geralmente satisfatório. Porém, quando a lesão cervical é grande, e são inevitáveis múltiplas aplicações da alça de alta freqüência, a conização a frio é preferivel, pois a transecção tecidual dificulta a avaliação da profundidade e largura da invasão estromal.

Assim, concluímos que, apesar de a colposcopia ser o método de escolha em face de um resultado citológico anormal, para orientar o local a ser biopsiado, suas restrições são sérias. Quando a lesão é microinvasora, o diagnóstico colposcópico é mais difícil, porque não existem achados característicos bem definidos para esta lesão. A excisão de lesões de alto grau por conização, independentemente dos achados clínicos e colposcópicos encontrados, é, portanto, justificada pela freqüente subavaliação de lesões de maior gravidade. A conização por CAF é método simples e de fácil execução, porém os critérios para sua indicação devem ser avaliados, pois apresenta maiores taxas de margens comprometidas e, conseqüentemente, maior risco de recidiva da doença em mulheres com NIC e maior freqüência de segundo procedimento em mulheres com carcinoma microinvasor.

\section{ABSTRACT}

Purpose: to determine the factors associated with the detection of a microinvasive carcinoma in the cervical cone of women with a previous colposcopically directed biopsy compatible with cervical intraepithelial neoplasia (CIN) 3 and to evaluate the proportion of involved margins.

Patients and methods: we reviewed the medical records of 385 women (mean age: 39 years) submitted to cold conization or conization by high frequency surgery (HFS) with a loop during the period from January 1993 to July 2000. These procedures were indicated on the basis of a biopsy compatible with (CIN) 3 .

Results: the diagnosis of the cone was compatible with (CIN) 3 in $243(63 \%)$ women and with (CIN) 2 in $13(3 \%)$. Only 10 presented HPV/CIN 1 (3\%) and eight had no residual disease in the cone. However, 101 (26\%) women presented a microinvasive carcinoma in the cone and $10(3 \%)$ presented a frankly invasive carcinoma. Age, menstrual status and number of deliveries were not related to the severity of the cone lesion. Women with oncologic colpocytology changes suggestive of invasion presented a significantly higher risk of having a microinvasive or invasive carcinoma as determined by final histology $(p<0.01)$, although 52 of the 243 women with CIN 2 or CIN 3 in the cone also showed a suggestion of invasion at colpocytology. Among the women with CIN 2 or 3, the epithelium was white in 44\%, dotted in $21 \%$, and mosaic-like in $17 \%$. This proportion was similar for women with a microinvasive or invasive carcinoma, with these images being detected in $37 \%, 23 \%$ and $21 \%$ of the cases, respectively. Involvement of the cone margins was significantly higher among women submitted to HFS (49\%) than among those submitted to cold conization (29\%).

Conclusion: the absence of independent clinical and colposcopic factors associated with the detection of a microinvasive carcinoma in women submitted to conization on the basis of a biopsy compatible with (CIN) 3 justifies the conical excision of the squamocolumnar junction in high grade cervical lesions.

KEY WORDS: Conization. Colposcopy. Intraepithelial neoplasia. Carcinoma, microinvasive.

\section{Referências}

1. Ang MS, Kaufman RH, Adam E, et al. Colposcopically directed biopsy and loop excision of the transformation zone: comparison of histologic findings. J Reprod Med 1995; 40:167-70.

2. Massad LS, Halperin CJ, Bitterman P. Correlation between colposcopically directed biopsy and cervical loop excision. Gynecol Oncol 1996; 60:400-3.

3. Prendiville W, Cullimore J, Norman S. Large loop excision of the transformation zone (LLETZ): a new method of management for women with cervical intraepithelial neoplasia. Br J Obstet Gynaecol 1989; 96:1054-60.

4. Lee KE, Koh CF, Watt WF. Comparison of the grade of CIN in colposcopically directed biopsies with that in outpatient loop electrosurgical excision procedure (LEEP) specimens: a retrospective review. Singapore Med J 1999; 40:694-6.

5. Denny LA, Soeters R, Dehaeck K, Bloch B. Does colposcopically directed punch biopsy reduce the incidence of negative LLETZ? Br J Obstet Gynaecol 1995; 102:545-8.

6. Duggan BD, Felix JC, Muderspach LI, et al. Coldknife conization versus conization by the loop electrosurgical excision procedure: a randomized, prospective study. Am J Obstet Gynecol 1999; 180:276-82.

7. Benedet JL, Bender H, Jones H 3rd, Ngan HY, Pecorelli S. FIGO staging classifications and clinical practice guidelines in the management of gynecologic cancers. Int $J$ Gynaecol Obstet 2000, 70:209-62. 
8. Hulman G, Pickles CJ, Gie CA, Dowling FM, Stocks PJ, Dixon R. Frequency of cervical intraepithelial neoplasia following large loop excision of the transformation zone. J Clin Pathol 1998; 51:375-7.

9. Narducci F, Occelli B, Boman F, Vinatier D, Leroy JL. Positive margins after conization and risk of persistent lesion. Gynecol Oncol 2000; 76:311-4.

10.Scully RE, Bonfiglio TA, Kurman RJ, Silverberg SG, Wilkins EJ. Histological typing of female genital tract tumors. 2nd ed. Berlin: Springler-Verlag; 1994.

11.Hopman EH, Kenemans P, Helmerhorst TJ. Positive predictive rate of colposcopic examination of the cervix uteri: an overview of literature. Obstet Gynecol Survey 1998; 53:97-106.

12.Ehsani N, Golbang P, Papadakis T, et al. The use of large loop excision of the transformation zone in management problems of cervical intraepithelial neoplasia Aust $\mathrm{N} \mathrm{Z} \mathrm{J} \mathrm{Obstet}$ Gynaecol 1998; 38:251-3.

13.Buxton EJ, Luesley DM, Shafi MI, Rollason M. Colposcopically directed punch biopsy: a potentially misleading investigation. Br J Obstet Gynaecol 1991; 98:1273-6.

14.Baldauf JJ, Dreyfus M, Ritter J, Philippe E. An analysis of the factors involved in the diagnostic accuracy of colposcopically directed biopsy. Acta Obstet Gynecol Scand 1997; 76:468-73.

15.Orlandi C, Costa S, Terzano P, et al. Presurgical assessment and therapy of microinvasive carcinoma of the cervix. Gynecol Oncol 1995; 59:255-60.

16.Reis AFF, Oliveira e Silva RJ, Camargo MJ, Silveira CMG. Validade da citologia e da biópsia orientada pela colposcopia no diagnóstico do carcinoma cervical pré-clínico. Rev Bras Ginecol Obstet 1999; 21:193-200.
17. Bethesda 2001. Proposed Bethesda 2001. Terminology: reflects these recommendations. [cited 2001 ago 24].Available from: URL:<http:/ / www.bethesda2001.cancer.gov.>

18.Choo YC, Chan OL, Hsu C, Ma HK. Colposcopy in microinvasive carcinoma of the cervix: an enigma of diagnosis. Br J Obstet Gynaecol 1984; 91:1156-60.

19.Benedet JL, Anderson GH, Boyes DA. Colposcopic accuracy in the diagnosis of microinvasive and occult invasive carcinoma of the cervix. Obstet Gynecol 1985; 65:557-62.

20. Carvalho JP. Estudo de fatores relacionados ao estado das margens cirúrgicas no tratamento das neoplasias intra-epiteliais cervicais escamosas de alto grau [dissertação]. São Paulo: Universidade de São Paulo; 2000.

21.Taha NSA, Focchi J, Ribalta JCL, Stávale JN, Dôres GB, Lima GR. Avaliação do grau e da extensão das alterações térmicas produzidas pela cirurgia de alta freqüência no colo uterino. Rev Bras Ginecol Obstet 2001; 23:47-51.

22.Chang DY, Cheng WF, Torng PL, Chen RJ, Huang SC. Prediction of residual neoplasia based on histopathology and margin status of conization specimens. Gynecol Oncol 1996; 63:53-6.

23.Tseng CJ, Liang CC, Lin CT, et al. A study of diagnostic failure of loop conization in microinvasive carcinoma of the cervix. Gynecol Oncol 1999; 73:91-5.

24.Gurgel MS, Bedone AJ, Andrade LA, Panetta K. Microinvasive carcinoma of the uterine cervix: histological findings on cone specimens related to residual neoplasia on hysterectomy. Gynecol Oncol 1997; 65:437-40.

\section{RBGO}

\section{éumapublicaçãoda}

FEBRASGO

equeaceitaartigos provenientes deginecologistas, obstetras e de outras especialidades. Portanto, publique!!! Mande já seu artigo para RBGO 